\title{
El surf en la educación formal: revisión sistemática Surfing in formal education: systematic review
}

*Mikel Pérez-Gutiérrez, **J osé M anuel Castanedo-Alonso, *Carlos Cobo-Corrales

*Universidad de Cantabria (España), **U niversidad de Barcelona (España)

\begin{abstract}
Resumen: el objetivo del artículo fue realizar una revisión de los trabajos que estudiaron la práctica del surf dentro de la educación formal para conocer las iniciativas desarrolladas y resumir las orientaciones pedagógicas para su aplicación. Para ello, se real izó una revisión sistemática en las bases de datos Dialnet, Eric, SciELO, Scopus yW eb of Science. Se recuperaron 12 documentos, publicados entre 2008 y 2018, desarrollados en cinco países diferentes y centrados en el surf, el bodyboard y el standup paddle. Dichos trabajos concluyeron que debe real izarse una aproximación progresivaal medio acuático y al surf en tres fases (centro educativo, piscina y playa), comenzando con la práctica del bodyboard por ser más fácil y menos peligrosa que el surf de tabla corta. Paralas sesiones de piscinay playa, serecomiendautilizar el tiempo del recreo paralos desplazamientos, ubicando las sesiones en lasúltimas horas de la jornada escolar. Los docentes de Educación Física que quieran implementar el surf en sus clases deberían adquirir conocimientos relacionados con la seguridad en el mar, con el equipamiento necesario parala práctica y con la propia técnica del surf.
\end{abstract}

Palabras clave: surf, educación formal, enseñanza, docentes, educación física, revisión sistemática.

\begin{abstract}
: the aim of this paper was to carry out a review of documents studying the surfing in formal education for discovering the programs developed and summarizing the pedagogical orientations for its application. Thus, a systematic review was performed in Dialnet, Eric, SciELO, Scopus and Web of Science databases. Twelve documents, published from 2008 to 2018, developed in five different countries and focused on surfing, bodyboarding and standup paddle, were retrieved. These documents concluded that a progressive approach to the aquatic environment and to surfing should be done in three phases (educational centre, swimming pool and beach), starting with the practice of bodyboard since it is easier and safer than shortboard surfing. The recess time should be used for travels in those sessions developed in the swimming pool and the beach. M oreover, these sessions should be scheduled in the last hours of the school day. Physical Education teachers willing to implement surfing in their sessions should gain knowledge about sea safety, surfing equipment and its own techniques.
\end{abstract}

Keywords: surfing, formal education, teaching, teachers, physical education, systematic review.

\section{Introducción}

En la actualidad, el surf es una actividad deportiva con un impacto social, cultural, económico y deportivo muy importante. Aunque el boom del surf comenzó en la década de 1960 (Booth, 2007; Kampion, 1997; Westwick \& N eushul, 2013), durante los últimos años ha sufrido un importante crecimiento en los ámbitos anteriormente mencionados. Es un deporte practicado por millones de personas a nivel mundial (Westwick \& Neushul, 2013) que forma parte del programa de los Juegos O límpicos deTokio 2020 (Tokyo 20200 rganising Committee, 2018). Además, movilizaun sector turístico y económico que tiene un impacto económico mundial de 50 billones de dólares (M cGregor \& W ills, 2016) y se ha convertido en un campo de conocimiento e investigación en crecimiento (Pérez-Gutiérrez \& Cobo-

\footnotetext{
Fecha recepción: 28-08-20. Fecha de aceptación: 03-02-21

Mikel Pérez-Gutiérrez

mikel.perez@ unican.es
}

Corrales, 2020).

El surf se presenta como una actividad con gran potencial educativo, fuertemente vinculado al área de Educación Física y con afinidad con otras áreas curriculares como Ciencias de la N aturaleza o Ciencias Sociales. La importancia y pertinencia de las actividades físicas en la naturaleza dentro del ámbito educativo ha sido explicada y corroborada por distintos autores (Caballero Blanco, 2012; Cañada Díaz \& GarcíaPatricio, 2003; M éndez-Giménez \& Fernández-Río, 2011; Peñarrubia Lozano, Guillén Correas, \& Lapetra Costa, 2016). Del mismo modo, las actividades acuáticas poseen un alto potencial educativo debido al desarrollo de habilidades y competencias que contribuyen al desarrollo integral del alumnado, así como al conocimiento y respeto del medio natural (Albarracín Pérez, 2018; López Prado, Álvarez García, \& Fernández-M iranda Pardilla, 2015; M oreno M urcia \& Gutiérrez San M artin, 1998; De Paula, 2018). En el caso concreto del surf, además es un medio idóneo para la construcción social del litoral, provocando procesos de cohesión territorial, 
cultural eidentitariaaltamentevalorados por las políticas comunitarias europeas (DeAlesi, 2009; Ford \& Brown, 2005; GonzálezTrueba, 2018; H umberstone \& Brown, 2015; Steinberg, 2001, 2014; Young, 1999; Zavalza Hough-Snee \& Sotelo Eastman, 2017).

Siguiendo un orden cronológico, la producción científica sobre surf ha sido analizada considerando distintas fuentes de información como Web of Science (WoS), Sport Discus, Science Direct, SciELO y Google Académico (Brasil, Ramos, \& Goda, 2013), WoS y Pubmed (Garrido, 2014), las revistas brasileñas (Gomes, SouzaN eto, Rojo, \& Silva, 2017) oWoSy Scopus (PérezGutiérrez \& Cobo-Corrales, 2020). A pesar de la variedad de disciplinas científicas desde las que se estudia y analiza el fenómeno del surf, como las Ciencias M édicas, las Ciencias de la Vida, la Sociología o la Historia entre otras, son pocos los artículos científicos que centran su atención en el ámbito educativo o la metodología de enseñanza (Brasil et al., 2013; Gomes et al. , 2017; Pérez-Gutiérrez \& Cobo-Corrales, 2020), por lo que el profesorado de Educación Física no cuenta con una fundamentación teórica clara para poder incluir esta actividad de aprendizaje, 0 algunas de sus variantes 0 modalidades, en las programaciones didácticas. Por ello, la iniciativa más común en los centros escolares consiste en contratar los servicios de distintas empresas dedicadas al turismo activo para realizar las llamadas «semanas azules», donde se fomenta la práctica de actividades acuáticas (Cabildo de Fuerteventura, 2020; IES Ribera del Arga, 2019; Liceo M onjardín, 2020), incluido el surf en algunas de ellas (IES La Corredoria, 2020; IES Las Llamas, 2019; Ribamontán al Mar, 2020). Esta opción palia algunos de los condicionantes que dificultan la incorporación de actividades físicas en el medio natural, como el surf, en contextos de educación formal. Algunos de los condicionantes son: la formación de los docentes, la programación y planificación previas a la actividad, la implicación de la comunidad escolar 0 el apoyo de los agentes educativos; destacando entre estos condicionantes la falta de apoyo de las administraciones educativas para fomentar y aportar formación permanente sobre estas actividades, la falta de recursos económicos y materiales, así como la organización escolar referente a horarios y reglamentos del centro (Dalmau Torres, Jiménez Boratia, Gómez Estebas, \& Gargallo Ibort, 2020; Fuentesal García \& Zamorano Sande, 2021).

Por lo tanto, existe un interés por la inclusión del surf en la educación formal y en las clases de Educación Física, derivado de la inserción social que ha tenido esta práctica deportiva a lo largo de los años (Brasil et al., 2013), pero los trabajos científicos existentes hasta el momento ofrecen visiones parceladas de su implementación en contextos educativos. Estadispersión del conocimiento dificulta que los docentes interesados en la práctica del surf en la educación formal puedan aplicar en sus clases las orientaciones pedagógicas de los diferentes trabajos existentes. El presente trabajo pretende abordar esta laguna de conocimiento.

Así, el objetivo de este artículo fue realizar una revisión sistemática de los trabajos que estudiaron la práctica del surf dentro de la educación formal. Específicamente, se determinaron las características de las investigaciones encontradas, el contexto de inclusión del surf en la educación formal y las orientaciones pedagógicas para su enseñanza. Este trabajo pretende ofrecer a los investigadores interesados, una perspectiva general de los trabajos científicos desarrollados en la educación formal sobre el surf, así como evidencias para la enseñanza del surf a docentes y técnicos deportivos.

\section{Metodología}

Se realizó una revisión bibliográficasistemática sobre los estudios relacionados con la aplicación del surf en el ámbito de la educación formal, siguiendo las recomendaciones propuestas por Cohen, Manion, \& M orrison (2007). Paraello, se buscaron todoslosartículos y revisiones relacionadas con dicha temática indexadas en las bases de datos Dialnet, Eric, SciELO, Scopus, SPORTDiscus y WoS. Se seleccionó la base de datos Dialnet por ser uno de los mayores portales bibliográficos del mundo de literatura científica hispana (Fundación Dialnet, 2020). ERIC es la principal base de datos sobre investigación e información educativa (IES, 2020), mientras que SciELO responde a las necesidades de comunicación científica en los países en desarrollo y particularmente enAmérica Latinay el Caribe (SciELO, 2019). Por su parte, Scopus y W oS son consideradas las principales bases de datos internacionales (ReverterMasia, Hernández-González, J ové-D eltell, \& LegazArrese, 2016; Pérez-Escoda, 2017; ). La recogida de información se desarrolló en marzo 2020.

\section{Objeto de estudio}

Se definió el surf como todas aquellas actividades deportivas con competición y reconocidas por la Asociación Internacional deSurfing, esto es, bodyboarding, longboard, shortboard, carreras y surf en standup paddle (SUP), para-surfing, bodysurfing, wakesurfing y cualquier 
otra actividad de deslizamiento sobre cualquier tipo de ola, o sobre mar en calma utilizando equipamiento para el deslizamiento sobre las olas (ISA, 2020). Además, Ios documentos debían referirse al surf como deporte, ya que en inglés este término se utiliza en otras áreas de conocimiento, como Ciencias de la Computación o del Medio Ambiente, para referirse a navegar por la red 0 los estudios relacionados con el océano y la costa. Finalmente, los documentos debían hacer referencia a la aplicación o estudio del surf dentro de la educación formal, entendida como aquella educación institucionalizada, intencional y planificada a través de organizaciones públicas y organismos privados reconocidos y que, en su totalidad, conforma el sistema educativo formal de un país. Es decir, que el surf se incluya dentro de al guna de las asignaturas 0 áreas que forman parte del sistema educativo (UNESCO, 2012). Teniendo en cuenta lo anterior, los criterios de inclusión fueron los siguientes:

- Artículos o revisiones indexadas hasta marzo de 2020 en las bases de datos Dialnet, Eric, SciELO, Scopus yWoS.

- Documentos que analicen el surf en cualquier nivel del sistema educativo formal, desde Educación Infantil hasta la Educación Superior (UNESCO, 2012).

- Documentos publicados en español, inglés 0 portugués.

\section{Estrategias de búsqueda}

En laWoS se seleccionaron las bases de datos Science Citation Index Expanded (SCI-EXPANDED), Social Sciences Citation Index (SSCI) y Arts \& Humanities Citation Index (A\&HCI). La búsqueda de documentos científicosse realizó en el título, palabras clavey resumen de los documentos, utilizando la búsqueda avanzada, los filtros por tipo de documento y losoperadores booleanos, para facilitar la recuperación de información. Además, se crearon dos cadenas de búsqueda, una en español y otra en inglés, para incluir los diferentes términos

Tabla 1

\begin{tabular}{ccc}
\multicolumn{3}{c}{ Términos y cadena de búsqueda utilizada para la recogida de información. } \\
\hline Español & Inglés \\
\hline Surf & Surf \\
Surfing & Surfing \\
Bodyboard & Bodyboard \\
Longboard & Longboard \\
Kneeboard & Kneeboard \\
"standup paddle" & "standup paddle" \\
& Paddleboard & Paddleboard \\
& Sup & Sup \\
\hline Deporte & deporte & sport \\
\hline Educación & educación & education \\
\hline & (surf OR surfing OR & (surf OR surfing OR \\
& bodyboard OR longboard OR & bodyboard OR longboard OR \\
kneeboard OR "standup & kneeboard OR "standup \\
Cadena de búsqueda & paddle" OR paddleboard OR & paddle" OR paddleboard OR \\
& sup) AND (deporte) AND & sup) AND (sport) AND \\
(educacion) & (education)
\end{tabular}

asociadosal surf, así como su delimitación como deporte y dentro del ámbito educativo (Tabla 1).

\section{Selección de documentos}

El proceso de búsqueda y selección de documentos aparece representado en laFigura 1. Los 469 documentos encontrados fueron filtrados, en primer lugar, por tipo de documento mediante los filtros de las bases de datos y posteriormente se exportaron al programa Endnote $X 9$. En segundo lugar, tras eliminar los documentos duplicados, se consultó el título y resumen de los restantes para determinar su vinculación con el objeto de estudio. Finalmente, fue necesario revisar el texto completo de 30 documentos para determinar su inclusión final.

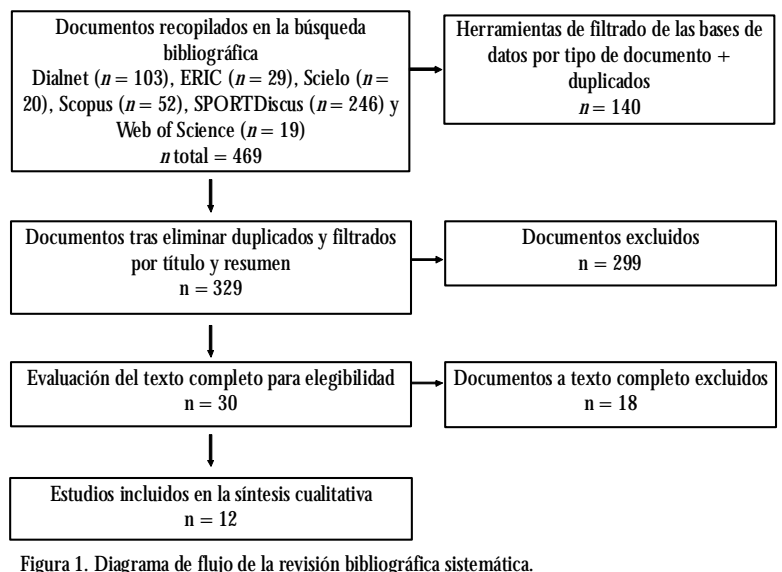

\section{Extracción y análisis de datos}

Tanto el proceso de selección como el análisis de documentos fue desarrollado por dos investigadores independientes y cuando hubo discrepancias entreambos se consultó a un tercer investigador. Mediante el análisis documental de contenido (Moreiro González, 2004; Pinto Molina, 2001), se realizó un resumen informativo de los documentos seleccionados atendiendo a su objetivo, metodología, principales resultados 0 conclusiones. Además, los documentos fueron categorizados atendiendo al tipo de metodología utilizada, el enfoque del estudio, la etapa educativadonde se desarrolló el estudio y su temática. Para determinar la metodología y enfoque del estudio se siguió la clasificación realizada por Hernández Sampieri, Fernández Collado y Baptista Lucio (2010). Respecto a la etapa educativa, se utilizaron los niveles educativos de la UNESCO (2012); mientras que la categorización de los trabajos según su temática se basó en el trabajo previo de Souza, Silva y M oreira (2016). 


\section{Resultados}

La presente revisión bibliográfica recuperó 12 artículos relacionados con la aplicación del surf dentro del ámbito de la educación formal, publicadosentre2008 y 2018, desarrollados en cinco países diferentes y centrados principalmente en la aplicación del surf con tabla corta por encima de otras disciplinas deportivas como el bodyboard o el standup paddle. Además, en estos trabajos predominó la aplicación de la metodología cualitativa (Tabla 2).

\begin{tabular}{|c|c|c|c|c|}
\hline Autores & $\begin{array}{l}\text { Afiliación autor } \\
\text { principal (país) }\end{array}$ & Revista & Modalidad & Metodología \\
\hline Bravo et al., 2016 & $\begin{array}{c}\text { California State } \\
\text { University San Marcos } \\
\text { (EE.UU.) }\end{array}$ & $\begin{array}{l}\text { Journal of Strength and } \\
\text { Conditioning Research }\end{array}$ & Surf & Cuantitativa \\
\hline $\begin{array}{l}\text { Cavanaugh et al., } \\
2013\end{array}$ & $\begin{array}{c}\text { Canisius College } \\
\text { (EE.UU.) }\end{array}$ & Palaestra & Surf* & Cualitativa \\
\hline $\begin{array}{c}\text { Cavanaugh \& } \\
\text { Rademacher, } 2014\end{array}$ & $\begin{array}{l}\text { Canisius College } \\
\text { (EE.UU.) }\end{array}$ & $\begin{array}{c}\text { The Journal of the } \\
\text { International Association of } \\
\text { Special Education }\end{array}$ & Surf & $\begin{array}{l}\text { Cuantitativa } \\
\text { Cualitativa }\end{array}$ \\
\hline $\begin{array}{l}\text { Clapham et al., } \\
2014\end{array}$ & $\begin{array}{l}\text { Univer sity of Rhode } \\
\text { Island (EE.UU.) }\end{array}$ & $\begin{array}{c}\text { Journal of Physical } \\
\text { Education, Recreation \& } \\
\text { Dance }\end{array}$ & Surf* & Cuantitativa \\
\hline $\begin{array}{l}\text { Clapham et al., } \\
2018 \\
\end{array}$ & $\begin{array}{l}\text { Univer sity of Rhode } \\
\text { Island (EE.UU.) }\end{array}$ & Palaestra & Surf* & Cuantitativa \\
\hline $\begin{array}{l}\text { Hignett et al., } \\
2018\end{array}$ & $\begin{array}{l}\text { Plymouth University } \\
\text { (Reino Unido) }\end{array}$ & $\begin{array}{c}\text { Journal of Adventure } \\
\text { Education and O utdoor } \\
\text { Learning }\end{array}$ & Surf & $\begin{array}{l}\text { Cuantitativa } \\
\text { Cualitativa }\end{array}$ \\
\hline $\begin{array}{l}\text { López Prado et al., } \\
2013\end{array}$ & $\begin{array}{l}\text { Consejería de Educación } \\
\text { del Principado de } \\
\text { Asturias (España) }\end{array}$ & $\begin{array}{c}\text { Revista Española de } \\
\text { Educación Físicay } \\
\text { Deportes }\end{array}$ & Surf* & Cualitativa \\
\hline $\begin{array}{l}\text { Machota Blas, } \\
2014\end{array}$ & $\begin{array}{l}\text { IESD. Antonio Hellín } \\
\text { Costa (España) }\end{array}$ & Cuadernos del profesorado & Bodyboard & Cualitativa \\
\hline $\begin{array}{l}\text { PérezTurpin et al., } \\
2008\end{array}$ & $\begin{array}{l}\text { Univer sidad de Alicante } \\
\text { (España) }\end{array}$ & $\begin{array}{l}\text { Agora para la educación } \\
\text { física y el deporte }\end{array}$ & Bodyboard & Cualitativa \\
\hline Ramos et al., 2013 & $\begin{array}{l}\text { Univer sidade do Estado } \\
\text { de Santa Catarina } \\
\text { (Brasil) }\end{array}$ & Revista da Educação Física & Surf* & Cualitativa \\
\hline Rynne, 2016 & $\begin{array}{c}\text { The Univer sity of } \\
\text { Q ueensland (Australia) }\end{array}$ & $\begin{array}{l}\text { Sport, Education and } \\
\text { Society }\end{array}$ & Surf & Cualitativa \\
\hline $\begin{array}{l}\text { Zagare } \& \text { Wuo } \\
\text { Pereira, } 2015\end{array}$ & $\begin{array}{l}\text { Univer sidade Nove de } \\
\text { Julho (Brasil) }\end{array}$ & $\begin{array}{l}\text { Lecturas: Educación física y } \\
\text { deportes }\end{array}$ & $\begin{array}{l}\text { Standup } \\
\text { Paddle }\end{array}$ & Cualitativa \\
\hline \multicolumn{5}{|c|}{ *: los autores utilizaron el término "surf" para referirse a la modalidad deportiva de tabla corta. } \\
\hline
\end{tabular}

A continuación, se categorizan los resultados encontrados atendiendo a la etapa educativa, enfoque, temática y razones que justifican la inclusión del surf en la educación formal, así como las orientaciones pedagógicas para dicha inclusión.

\section{Etapa educativa}

En los trabajos rescatados se analiza la práctica del surf en distintas etapas educativas, tal es como Educación Secundaria (Bravo, Cummins, N essler, \& N ewcomer, 2016; Cavanaugh \& Rademacher, 2014; Clapham, Lamont, Shim, \&Armitano, 2018; Hignett, W hite, Pahl, Jenkin, \& Froy, 2018; Machota Blas, 2014), Educación Primaria y Secundaria de manera conjunta (Cavanaugh, Rachmacher, Rachmacher, \& Simmons, 2013; Clapham, Armitano, Lamont, \& Audette, 2014; López Prado, Pardilla Fernández-M iranda, \& Álvarez García, 2013), Educación Post-Secundaria (Ramos, Brasil, \& Goda, 2013; Rynne, 2016) y Educación Terciaria (Pérez Turpin,
Chinchilla Mira, Suárez, Cortell \& Cejuela, 2008 ). Sólo el trabajo de Zagare y Wuo Pereira (2015) no indicó ninguna etapa educativa para el desarrollo de su propuesta, pudiendo ser aplicada a Educación Primaria o Secundaria.

\section{Enfoque}

Estos trabajos presentaron fundamentalmente: enfoques prácticos para evaluar los niveles de actividad física logrados en las clases de Educación Física en los que se implementaba el contenido de surf (Bravo et al., 2016); el impacto de un campamento de surf sobre las competencias social es y el autoconcepto de estudiantes con trastorno del espectro autista (Cavanaugh \& Rademacher, 2014); los efectos físicos y psicosociales de un programa de surf con personas con discapacidad (Clapham et al., 2014); los efectos fisiológicos de un programa de surf en un estudiante con trastorno del espectro autista (Clapham et al., 2018); el análisis de los efectos de un programa de surf de 12 semanas para estudiantes excluidos 0 en riesgo de exclusión escolar (Hignett et al., 2018); la contribución de un programa de surf local en la vida de jóvenes de comunidades indígenas (Rynne, 2016); o la construcción de una tabla de standup paddle con materiales reciclados (Zagare \& Wuo Pereira, 2015). Por su parte, los estudios teóricos estuvieron dirigidos a: la exposición de unidades didácticas de surf de tabla corta (López Prado et al., 2013) y de bodyboard (Machota Blas, 2014); el análisis de las percepciones de entrenadores respecto a los conocimientos pedagógicos para la enseñanza del surf (Ramoset al., 2013). Además, PérezTurpin et al. (2008) presentaron un enfoque mixto en el que se expuso la fundamentación teórica del aprendizaje situado junto con su desarrollo práctico aplicado al bodyboard, mientras que Cavanaugh et al. (2013) describieron el diseño e implementación de un campamento de surf para estudiantes con trastorno del espectro autista.

\section{Temática}

En este sentido, los documentos analizados estuvieron principalmente centrados en aspectos educativos o formativos (Cavanaugh et al., 2013; López Prado et al., 2013; M achota Blas, 2014; PérezTurpin et al., 2008; Ramos et al., 2013; Zagare \& Wuo Pereira, 2015), por encima de los aspectos psicológicos (Cavanaugh \& Rademacher, 2014; Clapham et al., 2014; Hignett et al., 2018). Además, también fueron tratados temas relacionados con el entrenamiento (Bravo et al., 2016; Clapham et al., 2018) y los aspectos sociales, 
culturales e históricos del surf (Rynne, 2016).

\section{Justificación de la inclusión del surf en la educación formal}

La justificación de la inclusión del surf dentro de la educación formal estuvo supeditadaal carácter novedoso de esta actividad (Bravo et al., 2016), sus beneficios físicosy psicológicos (Cavanaugh et al., 2013; Cavanaugh \& Rademacher, 2014; Clapham et al., 2014; Clapham et al., 2018; Hignett et al. , 2018), su potencial educativo para desarrollar el currículo de Educación Física (López Prado et al. , 2013; Machota Blas, 2014; PérezTurpin et al. , 2008; Zagare \&Wuo Pereira, 2015), su crecimiento como campo de intervención del profesional de Educación Física en Brasil (Ramos et al., 2013), y su relación con metassociales, educativasy desalud (Rynne, 2016).

\section{Orientaciones pedagógicas para la inclusión del surf en la educación formal \\ Losdocumentos anal izados propusieron las siguientes}

orientaciones pedagógicas:

(a) Utilizar el bodyboard antes que el surf debido a su facilidad de práctica, su menor peligrosidad y su disfrute desde el primer instante (Machota Blas, 2014).

(b) Enseñar de forma progresiva las habilidades propias del surf mediante situaciones de aprendizaje desarrolladas en el centro educativo, la piscina y la playa. En el centro educativo se presentan actividades con elementos inestables para el trabajo del equilibrio y al gunas de las habilidades propias del surf. En la piscina paratratar de simular el surf en un ambiente controlado y familiarizar al alumnado con el medio acuático y la adquisición de las habilidades específicas de la natación y del surf. En la playa para vivenciar la práctica del surf y desarrollar las habilidades adquiridas en las fases anteriores (López Prado et al., 2013).

(c) Utilizar materiales como el Bosu, Fitball, monopatín y similares para el trabajo del equilibrio (López Prado et al., 2013).

(d) Realizar sesiones prácticas en las últimas horas de la jornada escolar para aprovechar el tiempo de los

Tabla 3

Cuadro resumen de los documentos seleccionados.

\begin{tabular}{|c|c|c|c|c|}
\hline Autores & O bjetivo & Muestra & Instrumentos & Resultados \\
\hline $\begin{array}{l}\text { Bravo et al., } \\
2016\end{array}$ & $\begin{array}{c}\text { Analizar el papel del surf como estrategia para } \\
\text { aumentar los niveles de actividad física de los } \\
\text { jóvenes. }\end{array}$ & $\begin{array}{l}24 \text { estudiantes de instituto } \\
\text { (15-18 años) }\end{array}$ & Pulsómetros y videocámara & $\begin{array}{l}\text { La duración media de la clase fue de } 61.7 \text { minutos, estando activos } \\
\text { durante } 54.7 \text { minutos. Esperar a la olay remar ocuparon un } 42.7 \% \\
\text { y } 36.7 \% \text { del total del tiempo. }\end{array}$ \\
\hline $\begin{array}{l}\text { Cavanaugh et } \\
\text { al., } 2013\end{array}$ & $\begin{array}{l}\text { Presentar el diseño e implementación de un } \\
\text { campamento de surf de dos días para } \\
\text { estudiantes con trastorno del espectro autista. }\end{array}$ & $\begin{array}{l}21 \text { estudiantes (5-17 años) } \\
40 \text { familiares y } 26 \text { hermanos de } \\
\text { los estudiantes }\end{array}$ & Análisis descriptivo. & $\begin{array}{c}\text { Es una propuesta que pretende mejorar las habilidades sociales y } \\
\text { proporcionar experiencias multisensoriales a los estudiantes con } \\
\text { trastorno del espectro autista, así como incrementar su éxito y } \\
\text { compromiso activo en el aprendizaje. }\end{array}$ \\
\hline $\begin{array}{l}\text { Cavanaugh \& } \\
\text { Rademacher, } \\
\quad 2014\end{array}$ & $\begin{array}{l}\text { Deter minar los beneficios y consecuencias de } \\
\text { un campamento de surf de dos días sobre la } \\
\text { competencia social, habilidades sociales y } \\
\text { autoconcepto en estudiantes con trastorno del } \\
\text { espectro autista. }\end{array}$ & $\begin{array}{c}\text { Escuela } 1 \\
6 \text { estudiantes } 8 \text { familiares } \\
\text { (13.2 y } 46 \text { años) } \\
\text { Escuela } 2 \\
5 \text { estudiantes y } 10 \text { familiares } \\
\text { (12.6 y } 45.8 \text { años) } \\
28 \text { voluntarios } \\
\end{array}$ & $\begin{array}{l}\text { Social Skills Improvement System; Piers H Harris } \\
\text { Children's Self Concept Scale; Parent Perceptions of } \\
\text { the Surf Camp Curriculum; SURF Skills Checklist; } \\
\text { SURF Camp Curricul um Activity O bservation } \\
\text { Checklist. }\end{array}$ & $\begin{array}{l}\text { No existieron diferencias significativas en los resultados generales. } \\
\text { Cambios observables en la afirmación, responsabilidad, } \\
\text { compromiso, interacción y confianza. }\end{array}$ \\
\hline $\begin{array}{l}\text { Clapham et } \\
\text { al., } 2014\end{array}$ & $\begin{array}{l}\text { Examinar los efectos físicos y psicosociales de } \\
\text { un programa de surf con per sonas con } \\
\text { discapacidad. }\end{array}$ & $\begin{array}{l}17 \text { participantes } \\
\text { (5-17 años) }\end{array}$ & $\begin{array}{l}\text { Brockport Physical Fitness Test, pulsómetros y } \\
\text { monitores de actividad. }\end{array}$ & $\begin{array}{l}\text { Se observó que los participantes tenían mayor confianza en sí } \\
\text { mismos, mejoraron su desarrollo social interaccionando con otros y } \\
\text { estuvieron más relajados en el agua. }\end{array}$ \\
\hline $\begin{array}{l}\text { Clapham et } \\
\text { al., } 2018\end{array}$ & $\begin{array}{l}\text { Determinar las mejoras fisiológicas de un } \\
\text { programa de surf de } 8 \text { semanas en un } \\
\text { adolescente con trastorno del espectro autista. }\end{array}$ & $\begin{array}{l}1 \text { estudiante } \\
\text { (12 años) }\end{array}$ & Brockport Physical FitnessTest. & $\begin{array}{l}\text { El estudiante mejoró su capacidad aeróbica, fuerza muscular, } \\
\text { resistenciay rango de movimiento. }\end{array}$ \\
\hline $\begin{array}{l}\text { Hignett et al., } \\
2018\end{array}$ & $\begin{array}{l}\text { Anal izar los beneficios de un programa de surf } \\
\text { ' de } 12 \text { semanas sobre jóvenes excluidos o en } \\
\text { riesgo de exclusión escolar. }\end{array}$ & $\begin{array}{l}58 \text { estudiantes } \\
\text { (14.25 años) }\end{array}$ & $\begin{array}{l}\text { Frecuencia cardiaca y presión arterial. } \\
\text { UK Longitudinal Household Panel Survey; } \\
\text { 'Inclusion of Nature in the Self' scale; Entrevista } \\
\text { sobre el humor y actitudes hacia el surf; } \\
\text { Parent-Child Interaction System; Preguntas de } \\
\text { conocimiento medioambiental; Preguntas de } \\
\text { evaluación global del programa; Social and } \\
\text { Emotional Aspects of Learning questionnaire. }\end{array}$ & $\begin{array}{l}\text { Los resultados indicaron que el programa de surf resultó en una } \\
\text { mayor satisfacción con la apariencia, la conexión con amigos, } \\
\text { positividad hacia la escuelay el conocimiento medioambiental. }\end{array}$ \\
\hline $\begin{array}{l}\text { López Prado } \\
\text { et al., } 2013\end{array}$ & $\begin{array}{l}\text { Presentar una propuesta para incluir el surf en } \\
\text { la clase de Educación Física en Educación } \\
\text { Primariay Educación Secundaria } 0 \text { bligatoria. }\end{array}$ & $\begin{array}{l}\text { Educación Primaria y Educación } \\
\text { Secundaria } 0 \text { bligatoria }\end{array}$ & Análisis descriptivo. & $\begin{array}{l}\text { Es una propuesta que pretende fomentar la práctica de actividad } \\
\text { física en edades posteriores, utilizando un acercamiento al surf } \\
\text { (centro escolar, piscina, playa) para aumentar la satisfacción del } \\
\text { alumnado y una progresión más rápida en el aprendizaje. }\end{array}$ \\
\hline $\begin{array}{l}\text { Machota Blas, } \\
2014\end{array}$ & $\begin{array}{c}\text { Presentar una propuesta para incluir el } \\
\text { bodyboard en la clase de Educación Física en } \\
\text { Educación Secundaria } 0 \text { bligatoria. }\end{array}$ & $\begin{array}{l}\text { Alumnos de } 4 \text { ㅇ de Educación } \\
\text { Secundaria } 0 \text { bligatoria }\end{array}$ & Análisis descriptivo. & $\begin{array}{c}\text { Es una propuesta que pretende despertar el interés del alumnado } \\
\text { por el mar como actividad física al ternativa saludable y concienciar } \\
\text { por el cuidado del mar. }\end{array}$ \\
\hline $\begin{array}{l}\text { PérezTurpin } \\
\text { et al., } 2008\end{array}$ & $\begin{array}{l}\text { Comprobar la influencia de la } \\
\text { enseñanza situada en los aprendizajes del } \\
\text { bodyboard. }\end{array}$ & $\begin{array}{l}4 \text { estudiantes } \\
\text { (21 años) }\end{array}$ & Video grabación y entrevista. & $\begin{array}{c}\text { La enseñanza situada del bodyboard favoreció la adquisición de } \\
\text { conocimientos y procedimientos, siendo necesaria la participación } \\
\text { del docente como mediador. }\end{array}$ \\
\hline $\begin{array}{l}\text { Ramos et al., } \\
\quad 2013\end{array}$ & $\begin{array}{l}\text { Verificar la percepción de los entrenadores } \\
\text { respecto a los conocimientos pedagógicos } \\
\text { empleados para la enseñanza del surf. }\end{array}$ & $\begin{array}{l}11 \text { entrenadores } \\
\text { (28-52 años) }\end{array}$ & Entrevista semiestructurada. & $\begin{array}{l}\text { Preocupación de los entrenadores e importancia de los contenidos } \\
\text { relacionados con el mar, el equipamiento y los movimientos } \\
\text { técnicos. }\end{array}$ \\
\hline Rynne, 2016 & $\begin{array}{c}\text { Explorar la contribución de programas de surf } \\
\text { locales a la vida de jóvenes de comunidades } \\
\text { indígenas. }\end{array}$ & $\begin{array}{c}23 \text { participantes } \\
\text { (15-25 años) } \\
26 \text { trabajadores } \\
\text { (22-50 años) } \\
15 \text { miembros de la comunidad } \\
\text { (25-80 años) }\end{array}$ & $\begin{array}{l}\text { Entrevistas, cuestionarios, grupos focales, } \\
\text { notas de campo, fotografías, videos, } \\
\text { observación participante y análisis } \\
\text { documental. }\end{array}$ & $\begin{array}{c}\text { Aprendizajes relacionados con la reconexión espiritual con la tierra } \\
\text { y el océano, redesar rollo de un conocimiento cultural indígena y } \\
\text { específico del surf, y restablecimiento de lazos familiares y } \\
\text { comunitarios. }\end{array}$ \\
\hline $\begin{array}{l}\text { Zagare \& Wuo } \\
\text { Pereira, } 2015\end{array}$ & $\begin{array}{l}\text { Presentar una propuesta para incluir el Stand } \\
\text { o Up Paddle en la clase de Educación Física y } \\
\text { vincularlo al reciclaje de materiales. }\end{array}$ & Escuel a pública en Sao Paulo & Análisis descriptivo. & $\begin{array}{c}\text { Es una propuesta factible de ser implementada en la clase de } \\
\text { Educación Física, construyendo tablas de Stand U p Paddle con } \\
\text { gar rafas de plástico. }\end{array}$ \\
\hline
\end{tabular}


recreos en los desplazamientos hasta el medio natural (MachotaBlas, 2014).

(e) Colocar a los estudiantes siempre bajo el ángulo devisión del profesorado, junto con un trabajo en grupos reducidos (M achota Blas, 2014).

(f) U tilizar, al menos, un responsable adulto por cada alumno 0 alumna (Clapham et al., 2014; Clapham et al., 2018) o incluso dos personas adultas por cadaalumno 0 al umna (Cavanaugh et al., 2013) para las actividades destinadas a alumnado con discapacidad.

(g) Prestar especial atención para evitar laadquisición de valoresnegativos asociadosa la práctica del bodyboard (PérezTurpin et al., 2008).

(h) Formar al profesorado en los contenidos relacionados con la seguridad en el mar y con el equipamiento, así como los movimientos técnicos del surf (López Prado etal., 2013; MachotaBlas, 2014; Pérez Turpin et al., 2008; Ramos et al., 2013).

\section{Discusión}

El número de publicaciones científicas relacionadas con el surf dentro de la educación formal parece escasa al considerar la relevancia social de esta práctica deportiva. Así, el surf en sus distintas modalidades se ha convertido en un estilo de vida practicado por millones de personas en todo el mundo, con una estimación entre 17 y 35 millones de practicantes (SurferToday, 2018; Westwick \& Neushul, 2013), con 108 federaciones nacionales (ISA, 2020) y que ha alcanzado los Juegos O límpicos (Tokyo 20200 rganising Committee, 2018). Esta repercusión social ha hecho que el surf se vincule al ámbito de la educación formal en lugares como Hawái, California, Australia, Nueva Zelanda, Brasil, Portugal 0 España, entreotros(Australian Curriculum, 2020; Bravo etal. , 2016; Toporek, 2011; Cantabria, 2014; Das Chagas, 2017; M ondragon U nibertsitatea, 2020; Surfing New Zeland, 2020; U niversidad de Lisboa, 2020). Pero también ha contribuido a un aumento del interés de los investigadores por esta práctica deportiva, tal como reflejó el trabajo de Pérez-Gutiérrez \& Cobo-Corrales (2020) sobre la producción científica de surf indexada en laW 0 S hasta2017, en el que se encontraron un total de 318 documentos. A pesar de la relevancia social, deportiva y académica del surf, parecen escasas las publicaciones encontradas en la presente revisión. Sin embargo, estos resultados vienen a complementar las fuentes de conocimiento utilizadas por los entrenadores de surf para sus actividades de enseñanza, que suelen ser principalmente informales a través del intercambio deconocimiento con otrosprofesionales(Ramos, Brasil, \& Goda, 2012). Además, los resultados deberían ser interpretados con cautela. Por un lado, es probable que existan más documentos relacionados con el surf en el ámbito educativo publicados en otras bases de datos, tipos de documentos o idiomas y, por otro lado, probablemente se estén desarrollando proyectos 0 iniciativas relacionadas con esta temática, pero no son publicadas en revistas científicas. Tal es el caso, en el ámbito nacional, del proyecto de la ONG Dignidade Lugo (2013), trabajos publicadosen formato libro (Millán Gómez, 2010), en otros idiomas (Etxaniz Peña, 2003), prácticas educativas que no han sido publicadas (Castanedo-Alonso, Pérez-Gutiérrez, \& Gutiérrez, 2020) 0 trabajos académicos (M anuz San Miguel, 2018), entre otros. Por otra parte, debido a la amplia tradición surfera (Warshaw, 2010; Westwick \& N eushul, 2013) y al destacado papel investigador de Australia en el contexto internacional (Pérez-Gutiérrez \& CoboCorrales, 2020), futuras investigaciones deberían centrar su atención en analizar las iniciativas educativas relacionadas con el surf en dicho país y que no aparecen en la producción científica. Los datos de la Tabla 2 mostraron que los principales países encargados de investigar sobre el surf en la educación formal fueron Estados U nidos deA mérica y España, seguidos de Brasil, corroborando parcialmente los resultados de Brasil et al. (2013). Sin embargo, los autores más productivos de publicaciones científicas relacionadas con el surf estuvieron afiliados a universidades australianas (PérezGutiérrez \& Cobo-Corrales, 2020), lo que difiere sustancial mente con nuestros resultados.

Aunque la mayoría de trabajos encontrados estuvieron dedicadosal surf corroborando losresultados de Pérez-Gutiérrez \& Cobo-Corrales (2020), algunos autores utilizaron estetérmino de manera coloquial para referirse a la molidad deportiva de tabla corta (Cavanaugh et al., 2013; Clapham et al., 2018; Clapham et al., 2014; López Prado et al., 2013; Ramos et al., 2013). Sin embargo, los actuales trabajos podrían ser complementados con estudios dedicados al análisis de otras modalidades asociadas al surf como por ejemplo el longboard, parasurfing, bodysurfing 0 wakesurfing. Del mismo modo, la mayoría de trabajos utilizaron una metodología cualitativa propia del ámbito educativo, por lo que se debe reforzar la investigación cuantitativa en este campo de conocimiento.

La presente revisión mostró que se están desarrollando trabajos principalmente en las etapas de Educación Primaria y Secundaria, reflejando la 
mencionada repercusión social y deportiva del surf, convirtiéndose en un deporte motivante que contribuye al trabajo de habilidades, conocimientos, actitudes y valores, y que se relaciona con el currículo de dichas etapas educativas (Bravo et al., 2016; López Prado et al. , 2013; M achotaBlas, 2014; PérezTurpin et al. , 2008). Por el contrario, las etapas de educación postsecundaria y terciaria apenas recibieron atención. Estas etapas, junto con Educación Infantil donde no se encontró ningún trabajo, son lagunas de investigación que deberían ser reforzadas. Además, el enfoque de estos trabajos fue principalmente práctico, siendo necesario que se desarrollen más propuestas teóricas para fortalecer la fundamentación teórica para la implementación del surf en la educación formal. Debido al objeto de estudio del presente trabajo, la temática de los documentos encontrados estuvo centrada en aspectos relacionados con lametodología paraimplementar el surf en el ámbito educativo, así como determinar los beneficios psicológicos derivados de su práctica, respectivamente.

Los estudios analizados han demostrado algunos de los beneficios derivados del surf, como ser una actividad que puede cumplir con las recomendaciones de actividad física moderada-vigorosa para los jóvenes debido a sus requisitos fisiológicos y su carácter novedoso y divertido (Bravo et al. , 2016; M achota Blas, 2014), mejoras en la afirmación, responsabilidad, compromiso, interacción y confianza de estudiantes con trastorno del espectro autista (Cavanaugh et al., 2013; Cavanaugh \& Rademacher, 2014), mejoras observables en las relaciones sociales de estudiantes con discapacidad (Clapham, Armitano, Lamont, \& Audette, 2014) y mejoras fisiológicas en un estudiante con trastorno del espectro autista (Clapham et al., 2018), mejora de la autoestima, la conexión con otros compañeros y positividad hacia la escuela y el conocimiento medioambiental de alumnos en exclusión o riesgo de exclusión escolar (Hignett et al., 2018), o la adquisición deaprendizajes relacionados con lareconexión espiritual con latierray el océano, el desarrollo de un conocimiento cultural indígena y específico del surf y el restablecimiento de lazos familiares y comunitarios para un grupo de jóvenes indígenas (Hignett et al., 2018). Es cierto que la muestra de participantes utilizada en estos trabajos es baja y no permite generalizar los resultados encontrados, sin embargo, los hallazgos parecen indicar que el contacto con la natural eza, con otras personas (practicantes, monitores y monitoras o miembros de la comunidad) y con una actividad física novedosa y divertida con altos requerimientos fisiológicos, son características que justifican el potencial educativo del surf. Ser una actividad físicamente desafiante, practicada al aire libre en compañía de otras personas de forma amistosa, segura y divertida son características que convierten al surf en una actividad promotora de bienestar (Godfrey, Devine-W right, \& Taylor, 2015) y que favorece el desarrollo de habilidades para la vida como la perseverancia, el trabajo en equipo, el control emocional, la concentración, el liderazgo y la toma de decisiones(Santoset al. , 2020), mediadaspor lainfluencia de las características sociales, físicas y culturales de este deporte (Ellmer, Rynne, \& Enright, 2019).

Además, el surf puede relacionarse con contenidos transversales como el reciclaje de materiales (Zagare $\&$ Wuo Pereira, 2015), la conciencia y respeto medioambiental (Hignett et al., 2018; Machota Blas, 2014; PérezTurpin et al., 2008) o la cultura oceánica y el mar (Rynne, 2016). Estainterdisciplinariedad también fue resaltada por otros autores (Albarracín \& M orenoMurcia, 2011; A raújo, FerreiraeSilva, \& O liveira, 2018; Auger \& Mirtain, 2009). Sin embargo, es necesario que el profesorado preste una atención especial al proceso de enseñanza-aprendizaje del bodyboard para la adquisición de valores positivos y evitar valores poco deseados que están asociados a la historia de esta práctica deportiva como, por ejemplo, el consumo de alcohol (PérezTurpin et al., 2008).

Aunque no fueron recuperados en la presente investigación, a nivel nacional existen trabajos relacionados con la temática. Así, A guiar Vera (2008) presentó un conjunto de orientaciones generales para la inclusión del surf con tablacortaen lasclases deEducación Física, mientras que el trabajo de Machota Blas (2012) estuvo dedicado a presentar la planificación de unaunidad didáctica de bodyboard, incluyendo las mismas recomendaciones metodológicas expuestas en su posterior trabajo (Machota Blas, 2014). El trabajo de López Prado \& García Suárez (2013) describió los aspectos metodológicos para el desarrollo del tarp surf en el ámbito escolar, profundizando en las actividades que se pueden desarrollar en el centro educativo para la mejora del surf (López Prado et al. , 2013). Por su parte, el estudio de Auger \& Mirtain (2009), traducido del original en francés, expuso las orientaciones metodológicas generales para la práctica del bodyboard, bodysurf y surf en el ámbito escolar junto con la descripción de distintos ejemplos de actividades. Finalmente, el trabajo deFernández deGamboa(2014), siguiendo las recomendaciones de López Prado et al. (2013), realizó una propuesta de planificación de la 
enseñanza del surf de tabla corta en cuatro fases, desarrolladas en el centro educativo dos de ellas, en la piscina y en el medio natural, corroborando la importancia de esta orientación metodológica. A nivel internacional, Rosado, M esquita, Correia y Pereira (2008) señalaron las concepciones metodológicas de los entrenadores de surf para la enseñanza de este deporte.

\section{Conclusiones}

La presente revisión recuperó un total de 12 documentos publicadosentre 2008 y 2018 queanal izaron la práctica del surf en el ámbito de la educación formal, lo que demuestra el carácter incipiente de este campo de investigación en comparación con la producción científica internacional relacionada con el surf. Los trabajos analizados estuvieron principal mente dedicados a presentar propuestas para la inclusión del surf en el ámbito educativo, utilizando metodologías cual itativas con enfoque práctico. Respecto a la etapa educativa, destacaron las propuestas aplicadasen Educación Primaria y Secundaria, por lo que es necesaria más investigación en Educación Postsecundaria y Terciaria. La temática del resto de trabajos fue variada, dedicándose a analizar la percepción de los entrenadores y entrenadoras sobre los conocimientos pedagógicos necesarios para la enseñanza del surf, la contribución del surf al aumento de los niveles de actividad física de los jóvenes, los efectos del surf sobre alumnos con discapacidad 0 en riesgo de exclusión social y el impacto de un programa de surf sobre la vida de jóvenes indígenas.

La inclusión del surf en la educación formal estuvo justificada ampliamente por los estudios analizados, aludiendo a los beneficios físicos, psicológicos y sociales derivados de su práctica, concretamente para alumnos con al guna discapacidad, en riesgo de exclusión social 0 pertenecientes a culturas indígenas, su carácter novedoso, su potencial para desarrollar el currículo de Educación Física, así como su relación con metas sociales, educativas y de salud.

Respecto a las orientaciones pedagógicas para la implementación del surf en el ámbito educativo, se propuso una aproximación progresivaal medio acuático y al surf en tresfases, indicando que debería comenzarse con la práctica del bodyboard por ser una modalidad másfácil, menospeligrosay que posibilitamayor disfrute desde el primer momento. Los profesores y profesoras de Educación Física que quieran implementar el surf en susclases deberían adquirir ciertaformación, destacando la adquisición de conocimientos relacionados con la seguridad en el mar, con el equipamiento necesario para la práctica y con la propia técnica del surf. Además, los docentes y técnicos deportivos deben prestar atención al proceso de enseñanza-aprendizaje para evitar la adquisición de valores negativos asociados a la práctica del surf. Los trabajos también mostraron ejemplos para vincular la práctica del surf con temas transversales, enriqueciendo el proceso de enseñanza-aprendizaje y la interdisciplinariedad de laasignaturade Educación Física.

Futuros trabajos deberían desarrollar investigaciones empíricas sobre laenseñanzadel surf, analizando el papel de los actores involucrados y los propios programas de surf, para obtener datos y evidencias que posibiliten la discusión científica y la aplicación de los conocimientos extraídos sobre la enseñanza del surf. En este sentido, debe determinarse la idoneidad y aplicabilidad de las orientaciones metodológicas extraídas en el presente trabajo para las etapas de Educación Primaria 0 Secundaria.

\section{Referencias}

Australian Curriculum (2020). Structure. Recuperado el 24 denoviembrede 2020, deAustralian Curriculum website: https: / / ww w. austral iancurricul um. edu. au/ f-10curriculum/ health-and-physical-education/ structure/ Aguiar Vera, U. (2008). Los deportes acuáticos: el surf en el áreadeEducación Física. Lecturas: Educación física y deportes, - (126). Recuperado de https:/ / www.efdeportes.com/ efd126/ los-deportes-acuaticos-el-surf-en-el-ar ea-deeducacion-fisica. htm

Albarracín, A. \& \& M oreno-M urcia, J.A. (2011).Valoración de lainclusión de lasactividades acuáticasen Educación Física. Motricidad, European Journal of Human M ovement, 26, 123139. Recuperado de https:/ / www. eurjhm. com/ index. php/ eurjhm/ article/ view/ 264

Albarracín Pérez, A. (2018). Actividades acuáticas educativas escolares. Revista de Investigación en Actividades Acuáticas, 2(3), 1-2. doi:10.21134/ riaa.v2i3.1444

Araújo, L. M., Ferreirae Silva, V. A., \& O liveira, N. C. (2018). Relato de experiência: a pedagogia do surf na educação básica. Revista EDaPECI, 18(2), 158-163. Doi: 0.29276/ redapeci.2018.18.28546.158-163

Auger, M. . \& M irtain, T. (2009). «Surfear» sobrela ola: Unidad didáctica progresamos en surf. Revista de Educación Física: Renovar la Teoría y Práctica, - (116), 33-37.

Brasil,V.Z., Ramos,V., \& Goda, C. (2013). A produção científica sobresurf: uma análiseapartir das publicações entre20002011. Revista Pensar a Prática, 16(3), 619-955. doi: 10.5216/ rpp.v16i3. 19466

Booth, D. (2007). Surfing En D. Booth \& H. Thorpe (Eds.), Berkshire Encyclopedia of Extreme Sports (pp. 317-323). 
London: Berkshire Publishing Group.

Bravo, M. M., Cummins, K. M., Nessler, J. A. \& \& N ewcomer, S. C. (2016). Heart rate responses of high school students participating in surfing physical education. Journal of Strength and Conditioning Research, 30(6), 1721-1726. doi:10.1519/JSC.0000000000001263

Caballero Blanco, P. J. (2012). Potencial educativo de las actividades físicas en el medio natural: Actividades de colaboración simple. EmásF: revista digital de educación física, (19), 99-114. Recuperado de https:// emasf2. webcindario.com/ NUMERO_19_EM ASF. pdf

Cabildo de Fuerteventura(2020). Lasemanäazul 2018-2020. Recuperado de http:// www. cabildofuer. es/ documentos/ Deportes/ Escuelas_nauticas/ semanaazul/ proyecto_semana_azul_2018-2020.pdf

Castanedo-Alonso, J. M., Pérez-Gutiérrez, M ., \& Gutiérrez, P. (2020). Mar deFondo: Surfear con laoladelas evidencias científicas [Entradaen blog]. Proyecto Crece. Recuperado de https: / / proyectocrece. eldiariomontanes. es/ mar-defondo-surfear-con-la-ola-de-las-evidencias-cientificas/

CañadaDíaz, S. \& GarcíaPatricio, B. (2003). El medio natural. En M. L. Rivadeneyra Sicilia (Ed.), D esarrollo de la motricidad en los distintos contextos deportivos (pp. 225-251). Sevilla: Wanceulen.

Cavanaugh, L. K., Rachmacher, S. , Rachmacher, J., \& Simmons, R. (2013). Planning and implementing a surf camp for students with autism spectrum disorder. Palaestra, 27(1), 17-22.

Cavanaugh, L. K., \& Rademacher, S. B. (2014). How aSU RFing social skills curriculum can impact children with autism spectrum disorders. The Journal of the International Association of Special Education, 15(1), 27-35. Recuperado de https: / / www. iase. org/ Publications/ JIASE\%202014\%20Edition. pdf

Clapham, E. D. , Armitano, C. N. , Lamont, L. S. \& \& Audette, J. G. (2014).Theocean asauniquetherapeutic environment: Developing asurfing program. Journal of Physical Education, Recreation \& Dance, 85(4), 8-14. doi:10.1080/ 07303084.2014 .884424

Clapham, E. D., Lamont, L. S., Shim, M., \& Armitano, C. (2018). A Case Report Illustrating the Implementation of aTherapeutic Surfing Intervention for an Adolescent with Autism. Palaestra, 32(2), 49-53.

Cohen, L., Manion, L., \& Morrison, K. (2007). M etaranalysis, research syntheses and systematic reviews. En L. Cohen, L. Manion, \& K. Morrison (Eds.), Research Methods in Education (pp. 335-343). London: Routledge.

DalmauTorres, J. M ., Jiménez Boratia, R., GómezEstebas, N., \& Gargallo lbort, E. (2020). Diagnóstico escolar sobre el tratamiento de las actividades físicas en el medio natural dentro de la asignatura de Educación Física. Retos: N uevas Tendencias en Educación Física, Deporte y Recreación, - (37), 460-464. doi: 10.47197/ retos. V37i37.71010

Decreto que establece el currículo de Educación Primaria en la Comunidad Autónoma de Cantabria 27/ 2014, 29, B. O.C. extraordinario, $\S 8557$ (2014).

Das Chagas, L. G. (2017). 0 surfe como tema da Educação Física em escolas públicas de Florianópolis/ SC. (Trabajo para la obtención del título de Licenciatura em Educação Física), Universidade Federal de Santa Catarina, Florianópolis. Recuperado de https:/ / repositorio. ufsc. br/ handle/ $123456789 / 177633$

DeAlesi, M. (2009). The customs and culture of surfing, and an opportunity for anew territorialism? Reef Journal, 1(1), 85-92. Recuperado dehttp:/ / thereefjournal.com/ files/ 6._De_Alessi.pdf

De Paula, L. (2018). Adaptación de los contenidos de la educación física a las actividades acuáticas en el ámbito escolar. Revista de investigación en actividades acuáticas, 2(3), 37-53. Recuperado de https:// revistas. innovacionumh.es/index.php/ investigacionactividadesacuatica/ article/ view/ $402 / 752$

Ellmer, E., Rynne, S. \& Enright, E. (2019). Learning in action sports: A scoping review. European Physical Education Review, 26(1), 263-283. doi: 10.1177/ 1356336X19851535

Etxaniz Peña, L. (2003). Surfa: Fitxa didaktikoak. Guipúzcoa, España: U rola Kostako U dal Elkartea.

Fernández de Gamboa, I. (2014). El surf como contenido en la asignatura de educación física: Propuestade inclusión y guía parael docente(Trabajo Fin de Máster). U niversidad Internacional deLaRioja, Vitoria-Gasteiz. Recuperado de https: / / reunir. unir. net/ bitstream/ handle/ $\begin{array}{lllllllllllllll}1 & 2 & 3 & 4 & 5 & 6 & 7 & 8 & 9 & / & 2 & 6 & 4 & 9 & /\end{array}$ fernandez\%20de\%20gamboa. pdffsequence $=1$ \&isAllowed $=y$ Ford, N., \& Brown, D. (2005). Surfing and social theory: Experience, embodiment and narrative of the dream glide. $\mathrm{New}$ York: Routledge.

Fuentesal García, J., \& Zamorano Sande, D. (2021). Incorporación de contenidos de actividades en el medio natural y de ocio en la educación formal (TSEAS): Una experiencia de espeleología (Incorporation of contents of activitiesin the natural environment and leisurein formal education (TSEAS): a caving Retos: Nuevas Tendencias en Educación Física, D eporte y Recreación, -(39), 820-828. doi: https: / / doi. org/ 10.47197/ retos. v0i39.80157

Fundación Dialnet (2020). Dialnet. Recuperado el 24 de noviembre de 2020, de Fundación Dial net website: https:/ / fundaciondialnet.unirioja. es/ dial net/ dialnet/

Garrido, N. (2014). Na 0 nda da Ciência. M otricidade, 10(4), 1-2. doi: $10.6063 /$ motricidade. 10(4).6256

Godfrey, C., Devine-W right, H., \& Taylor, J. (2015). The positive impact of structured surfing courses on the wellbeing of vulnerable young people. Community Pract, 88(1), 26-29. PMID: 26357740.

Gomes, L. do C., SouzaN eto,A., Rojo, J. R. , \& Silva, M. M. e. (2017). Nas O ndas da Ciência: Perfil da Produção do Conhecimento Sobre o Surf em Periódicos Brasileiros. LICERE - Revista Do Programa De Pós-graduação 
Interdisciplinar Em Estudos Do Lazer, 20(4), 285-311. doi: 10.35699/ 1981-3171.2017.1734

GonzálezTrueba, J. J. (2018). El turismo de surf en España: Nuevo recurso estratégico, impacto cultural y retos de sostenibilidad. ABACO, 23-54.

Hernández Sampieri, R., Fernández Collado, C. , \& Baptista Lucio, M. d. P. (2010). Metodología de la Investigación. México: McGraw-Hill.

Hignett, A. , W hite, M. P., Pahl, S., Jenkin, R., \& Froy, M. L. (2018). Evaluation of a surfing programme designed to increase personal well-being and connectedness to the natural environment among 'at risk' young people. Journal of Adventure Education and Outdoor Learning, 18(1), 53-69. doi:10.1080/ 14729679.2017.1326829

Humberstone, B. , \& Brown, M. (2015). Seascapes: Shaped by the sea. U nited Kingdom: Ashgate Publishing.

IES (2020). What isERIC?Recuperado el 24 de noviembre de 2020, de Institute of Education Sciences website: https:/ / ies.ed.gov/ ncee/ projects/ eric. asp

IES LaCorredoria(2020). Semana Azul'19. Recuperado el 30 de noviembre de 2020 de IES La Corredoria website: https: / / alojaw eb. educastur.es/ web/ ieslacorredoria/ depart/ ef/ activ/ semana-azul

IES Las Llamas (2019). Semana azul 2018. Recuperado el 28 de noviembre 2020 de IESLaAlbericia website: https: / / ieslasllamas.es/ index. php/ informacion-general/ historico-cursos/ 71-curso-2018-2019/ 141-semanaazul-2018

IES Ribera del Arga (2019). Semana azul 2019. Recuperado dehttps: / / iesriberadelarga. educacion. navarra.es/ web1/ blog/ 2019/ 05/ 29/ semana-azul-2019/

ISA (2020). About ISA. Recuperado el 20 de enero de 2021, de International Surfing Association website: https:/ / www. isasurf. org/ isa-info/ isa/

Kampion, D. (1997). Stocked: A history of surf culture. Koln: Evergreen.

Souza, D. L., Silva, M. M ., \& M oreira,T. S. (2016). 0 perfil da produção científica online em Português relacionada às modalidades olímpicas e paralímpicas. M ovimento, 22(4), 1105-1120. doi: 10.22456/ 1982-8918.64591

Liceo M onjardín (2020). SemanaAzul. Recuperado el 24 de noviembre de 2020, de Liceo M onjardín website: https:/ / liceomonjardin. net/ semana-azul.html

López Prado, J., Álvarez García, D. \& \& Fernández-M iranda Pardilla, S. (2015). Actividadesen el medio natural acuático en educación física. Valoración del profesorado sobre los factores que limitan su inclusión. EmásF: Revista Digital de Educación Física, - (33), 97-110. Recuperado de http:/ / e $\mathrm{m}$ a sf. w e b c i n d a r i o.co m / Actividades_en_el_medio_natural_acuatico_en_EF.pdf López Prado, J., \& G García Suárez, C. N. (2013). Propuesta de acercamiento del surf a los centros educativos: El tarp surf. EmásF: Revista Digital de Educación Física, - (21), 816. Recuperado de http:/ / emasf. webcindario.com/
El_tarp_surf.pdf

López Pradō, J., Pardilla Fernández-M iranda, S. \& Álvarez García, D. (2013). N uevos contenidosen educación física: orientaciones didácticas para la integración del surf en el medio educativo. Revista Española de Educación Física y Deportes, - (402), 93-106. Recuperado de https:/ / www. reefd. es/ index. php/ reefd/ article/ view/ 68/ 69

M achotaBlas, V. E. (2012). El bodyboard en el marco escolar: Una propuesta de aplicación. Emásf: Revista Digital de Educación Física, 4(19), 39-49.

M achota Blas, V. E. (2014). El surf en laEducación Secundaria: Una propuesta práctica. Espiral. Cuadernos del profesorado, 7(15), 42-48. Recuperado de http:// e $\mathrm{m}$ a s f. w e b c i n d a r i o.co m / El_Bodyboard_en_el_marco_escolar.pdf

Manuz San Miguel, Í. (2018). El surf en el ámbito educativo: Revisión bibliográfica (Trabajo de fin de grado). Universidad de Cantabria, Santander. Recuperado de http:/ / repositorio. unican. es: 8080/ xmlui/ bitstream/ $\mathrm{h}$ a $\mathrm{n}$ d l e / $100902 / 15200$ / M anuzSanM iguellñigo. pdf?sequence= 1 \&isAllowed=y

M cG regor, T., \&W ills, S. (2016). N atural assets: Surfing a wave of economic growth. Working Papers, U niversity of Sidney, School of Economics. Recuperado de http:/ / econwpseries.com/ 2016/ 201606.pdf

Méndez-Giménez, A. , \& Fernández-Río, J. (2011). Nuevas tendencias metodológicas en la enseñanza del esquí: 0 rientaciones didácticas para su iniciación en los centros educativos. Apunts. Educación Física y Deportes, - (105), 3543. doi: 10.5672/ apunts.2014-0983.es. (2011/ 3). 105.04

Millán Gómez, D. (2010). El surf: Propuesta didáctica para la ESO. Granada: PLANETBuk.

Moreiro González, J. A. (2004). El contenido de los documentos textuales: Su análisis y representación mediante el lenguaje natural. Gijón:Trea.

Moreno Murcia, J. A. , \& Gutiérrez San Martin, M. (1998). Bases metodológicas para el aprendizaje de las actividades acuáticas educativas. Barcelona: INDE.

Mondragon Unibertsitatea (2020). Curso Experto en Marketing y Management del Surf y Deportes Deslizantes. Recuperado el 4 de diciembre de 2020, de M ondragon U nibertsitatea Formación para Profesionales website: www. mondragon.edu/ cursos/ es/ tematicas/ direcciongestion-empresas-organizaciones/ curso-experto-enmarketing-y-management-del-surf-y

O N G Dignidade Lugo (2013). Agar Agar Surfing Club: Surf para el Cambio. En C. Solla Salvador (Ed.), Guía de Buenas Prácticasen Educación Inclusiva (pp. 112-116). Madrid: Save the Children.

Peñarrubia Lozano, C. , Guillén Correas, R. , \& LapetraCosta, S. (2016). Lasactividadesen el medio natural en educación física, ¿teoría o práctica?Cultura, Ciencia y D eporte, 11(31), 27-36. Recuperado de https:// 
pdfs. semanticscholar.org/ 0 b $24 /$ a 07 ce 0558 b $0 c 931$ f 9 e 782 f 096 cc 5 e 3 a0a9d7.pdf? ga $=2.212744913 .431092794 .1611617782-$ 348577923. 1611617782

Pérez-Escoda, A. (2017). WOS y SCOPU S: Ios grandes aliados de todo investigador. Recuperado el 27 de octubre de 2020, de Revista Comunicar website: https:/ / www. revistacomunicar.com/wp/ escuela-de-autores/ wos-y-scopus-los-grandes-aliados-de-todoinvestigador/

Pérez-Gutiérrez, M., \& Cobo-Corrales, C. (2020). Surfing scientific output indexed in theW eb of Science and Scopus (1967-2017). M ovimento: Revista da Escola de Educação Física, 26, e26015. doi:10.22456/ 1982-8918.94062

PérezTurpin, J.A., Chinchilla, J. J., Suárez, C., Cortell, J. M. . \& Cejuela, R. (2008). Aprendizaje situado en los deportes del mar: Bodyboard. Ágora para la educación física y el deporte, (7-8), 125-144. Recuperado de https:/ / rua.ua.es/ dspace/ bitstream/ 10045/ 23235/ 1/ 2008_Perez_Turpin_Agora.pdf

Pinto Molina, M. (2001). El resumen documental: Paradigmas, modelos y métodos. Madrid: Fundación Germán Sánchez Ruipérez.

Ramos, V., Brasil, V. Z., \& Goda, C. (2012). A aprendizagem profissional na percepção de treinadores de jovens surfistas. Revista da Educação Física / UEM, 23(3), 431442. doi: 10.4025/ reveducfis. v23i3.15320

Ramos, V., Brasil, V. Z. \& \& Goda, C. (2013). 0 conhecimento pedagógico parao ensino do surf. Revista da Educação Física, 24(3), 381-392. doi:10.4025/ reveducfis. V24.3.18730

Reverter-Masia, J., Hernández-González, V., Jové-D eltell, C., $\&$ Legaz-Arrese, A. (2016). Faculty-officials' web of science and scopus production with 6 years in sports science in Spain. Revista Interamericana de Bibliotecologia, 39(2), 149-162. doi: 10.17533/ udea.rib.v39n2a06

Ribamontán al Mar (2020). «Semana azul» del surf para centros escolares. Recuperado el 27 de octubre de 2020, de Surf a todo Costa website: http:/ / www. surfatodacosta.es/ semana-azul/

Rosado, A. F. B., Mesquita, I. M. R., Correia, M. E., \& Pereira, F. M. (2008). Concepções dos treinadores sobre o ensino do Surf no âmbito do Desporto Infanto-juvenil. Comunicación presentada al XII Congresso de Ciências do Desporto e Educação Física dos Países de Língua Portuguesa, Porto Alegre.

Rynne, S. (2016). Exploring the pedagogical possibilities of indigenous sport-for-development programmes using a socio-personal approach. Sport, Education and Society, 21(4), 605-622. doi:10.1080/ 13573322.2015.1107830

Santos, F., Bean, C. ,Azevedo, N., Cardoso, A., Pereira, P., \& Cruz, H. (2020). M oving From an Implicit to an Explicit Approach of Life Skills Development and Transfer: The Case of Surfing in Schools. SAGE Open, 10(2). doi: 10.1177/ 2158244020933316
SCiELO (2019). SciELO : Modelo de publicação eletrônica para países em desenvolvimento [online]. Recuperado de https:/ / wp.scielo.org/wp-content/ uploads/ Modelo SciELO.pdf

Surfing N ew Zeland (2020). Wave warriors. Recuperado el 4 de diciembre de 2020, de Surfing New Zeland website: http:/ / www. surfingnz.co.nz/ wave-warriors/

Steinberg, P. E. (2001). The social construction of the ocean. Cambridge: Cambridge U niversity Press.

Steinberg, P. E. (2014). Foreword on thalassography. En J. Anderson \& K. Peters (Eds.), Water Worlds: Human geographies of the 0 cean (pp. xiii-xv). Farnham, UK:A shgate.

SurferToday (2018). How many surfers are there in the world? Recuperado el 6 de diciembre de 2020, de SurferToday website: https: / / www. surfertoday. com/ surfing/ 14228how-many-surfers-are-there-in-the-world

Tokyo 2020 Organising Committee (2018). Olympic Sports: Surfing, about this sport. Recuperado el 6 de diciembre de 2020, deTokyo 2020 website: https: / tokyo2020.org/ en/ games/ sport/ olympic/ surfing/

Toporek, B. (2011). Surfing to become official school sport in Hawaii. Recuperado de EducationWeek website: https:/ / blogs. edweek.org/ edweek/ schooled_in_sports/ $\begin{array}{llllllll}2 & 0 & 1 & 1 & / & 1 & 0 & \text { / }\end{array}$ surfing_to_become_official_school_sport_in_hawaii.html UNESCO (2012). International Standard Classification of Education, ISCED 2011. M ontreal: UNESCO Institute for Statistics. Recuperado de http:/ / uis. unesco.org/ sites/ default/ files/ documents/ international-standardclassification-of-education-isced-2011-en. pdf

Universidad de Lisboa(2020). Post-Graduation H igh Performance Surf Coaching. Recuperado el 4 de diciembre, de Facultad de M otricidad Humana U niversidad de Lisboa website: http:/ / posgrad.fmh. ulisboa. pt/ surfcoaching/

Warshaw, M. (2010). The history of surfing. San Francisco: Chronicle Books.

Westwick, P., \& N eushul, P. (2013). The world in the curl: An unconventional history of surfing. New York: Crown.

Young, M. (1999). The relationship between tourist motivations and the interpretation of place meanings. Tourism Geographies, 1(4), 387-405. doi: 10.1080/ 14616689908721333

Zagare, T., \& Wuo Pereira, D. (2015). 0 stand up paddle adaptado às aulas de Educação Física. Lecturas: Educación física y deportes, - (202). Recuperado de https:/ / ww w. efdeportes. com/ efd202/ 0-stand-up-paddleadaptado-de-educacao-fisica. htm

ZavalzaH ough-Snee, D. \& Sotelo Eastman, A. e. (2017). The critical surf studies reader. London: Duke U niversity Press. 